\title{
La hausse conjointe des prix de l'énergie et des prix agricoles entre 2006 et 2008 : la spéculation et les biocarburants sont-ils coupables ?*
}

\author{
Tancrède VOITURIEZ \\ CIRAD, chercheur associé à I'Institut \\ du développement durable et des relations \\ internationales, 27 rue Saint-Guillaume \\ 75337 Paris Cedex 07, France \\ $<$ tancrede.voituriez@iddri.org>
}

\begin{abstract}
We review in this study the different factors which have been proposed in the literature as possible explanations of commodity prices sudden upsurge between 2006 and 2008. We examine as to whether scientific evidence validates any causal relationship. We emphasize in particular the role of explanatory variables underpinning the co-movement of energy and food price rise. Our aim is to provide an up-to-date understanding of food and energy market relationships, so as to better anticipate the possible changes in prices behaviour in the coming years.
\end{abstract}

Key words: energy prices, agricultural prices, food crisis, speculation
La hausse des prix des matières premières entre 2006 et 2008 a occupé dans les médias et les débats publics une place que ces produits, pris dans leur ensemble, avaient été dans l'impossibilité de reconquérir après les flambées historiques des cours en 1974 et 1979, concomitantes aux deux chocs pétroliers. Si les mouvements de prix sur les marchés avaient été chroniqués depuis lors, c'est surtout parce que ceux-ci, en apparence, inexorablement baissaient, au point d'apparaître comme un fléau endémique pour les économies et les ménages dont les revenus en dépendaient. Après la parenthèse euphorique des années 1970, le monde et en particulier le monde en développement redécouvraient au tournant du siècle les tourments de l'excès. Le pétrole lui-même n'échappait pas à la malédiction de l'abondance et pour reprendre un titre de The Economist resté fameux depuis, à l'inexorable déclin de ses prix sur les marchés ${ }^{1}$.

La crise de 2006-2008 a reçu dans ce contexte un écho particulier. Coup de tonnerre dans un ciel maussade, elle a surpris par son ampleur et sa soudaineté. L'indice des prix des matières premières mesuré par le $\mathrm{FMI}$, étalonné à la valeur 100 en 2005, monte à 130 en juillet 2006, dépasse 157 en novembre 2007 ; en juillet 2008, en moins de trois ans donc, il a plus que doublé, et s'établit à 219. II valait tout juste 60 , en moyenne, depuis 1992,

\footnotetext{
${ }^{*}$ Cet article est tirée d'une étude commandée et publiée par l'IFRI.

${ }^{1}$ Voir les articles "Drowning in oil" et "The next shock" de The Economist, 4 mars 1999 dans lesquels le journal britannique annonce que le prix du baril pourrait passer sous les $10 \$$.
}

année de sa création, jusqu'à 2004. Comment un tel phénomène n'a-t-il pu être anticipé par les politiques, pris au dépourvu par ses conséquences sur le revenu disponible, le pouvoir d'achat, l'inflation, et par le marché lui-même, comme incapable d'anticiper la rareté ? Les ressorts de l'ajustement entre l'offre et la demande sont-ils si dissimulés et si mystérieux pour que sur des marchés constitués depuis plus d'un siècle pour la plupart d'entre eux, des cours stables et déclinant en moyenne pendant plus de quinze ans se mettent à doubler ou tripler en valeur en l'espace de 30 mois? La clef des réponses à ces questions, dont les enjeux dépassent la seule recherche et touchent à la stabilité des économies et des relations internationales, réside dans l'identification des causes. Causes conjoncturelles et structurelles, causes propres ou externes à ces marchés, causes communes ou spécifiques aux produits alimentaires, boissons, produits agricoles industriels, métaux, énergie, qui composent le groupe des matières premières, de nombreux articles de presse et de science, de nombreux discours ont été écrits qui le plus souvent prennent position pour les unes contre les autres, qui plus rarement tentent de démêler les unes et les autres, sous ces différents qualificatifs, afin $d^{\prime}$ anticiper l'évolution longue des marchés.

Nous examinons dans cet article les différents facteurs qui ont pu être avancés en explication de la hausse des cours des matières premières entre 2006 et 2008, et les confrontons aux connaissances scientifiques disponibles à cette date. Dans cet exercice nous insistons en particulier sur les facteurs responsables des mouvements conjoints de cours sur les marchés de produits alimentaires et les marchés de l'énergie. Sous-jacent à notre travail figure le souhait de mieux comprendre les relations d'équilibre et de déséquilibre nouées entre ces deux marchés, afin de mieux anticiper leur changement possible durant ces dix prochaines années.

Nous rappelons dans la première partie les ordres de grandeur de la hausse des cours, que nous comparons dans le temps et entre grands groupes de produits. Nous abordons dans la seconde partie la question des mouvements conjoints de prix et des corrélations entre marchés. Nous établissons et commentons dans la troisième partie les causes les plus couramment avancées à la hausse des cours de matières premières, que nous essayons de classer selon l'importance des controverses qu'elles ont suscitées. Nous examinons dans la partie suivante trois causes particulièrement polémiques des mouvements conjoints de prix sur les marchés agricoles et de l'énergie, à savoir la hausse de l'offre de biocarburants, la transition alimentaire et énergétique des pays émergents, et la spéculation. Nous esquissons en conclusion les nouveaux équilibres sur lesquels ces relations entre marchés, entrevues durant l'épisode 2006-2008, pourraient durablement déboucher.

\section{La hausse des cours des matières premières, 2006-2008}

Plusieurs organismes, entreprises ou institutions publient des indices agrégés des prix de matières premières, ainsi de The Economist, du FMI, du Commodity Research Bureau (CRB), de la banque d'investissement Goldman Sachs ou encore du Hamburgishces WeltWirtschafts Institut (HWWI). Comme le souligne Luc Guyau [1] 
dans son rapport au Conseil économique, social et environnemental, ces indices diffèrent essentiellement par le nombre des matières premières retenues et les pondérations affectées à chacune avant agrégation. L'indice des prix du HWWI est jugé pertinent pour les pays développés, en raison du poids affecté aux matières premières énergétiques et industrielles, prépondérantes dans le panier moyen d'importation de ces pays. Nous privilégions ici l'emploi de l'indice du FMl, plus représentatif des produits d'intérêt pour les importateurs autant que pour les exportateurs de matières premières. Révisé au cours du temps pour tenir compte de l'évolution des proportions de chaque produit dans le commerce mondial, il repose aujourd'hui sur les volumes d'échange constatés en 2002-2004 (contre 1995-1997 dans sa version précédente). Le tableau 1 rend compte des pondérations affectées à chaque grand type de produits.

L'indice agrégé du prix des matières premières du FMI, créé en 1992, a suivi une évolution relativement stable durant les dix premières années de son existence, avant de connaître une hausse en 2002, soutenue à partir de 2004, culminant en juillet 2008, à laquelle a succédé un net reflux (figure 1). L'indice a triplé en valeur nominale entre janvier 2004 et juillet 2008. Sa moyenne durant les trois années de «boom » (2006-2008) s'établit à $130 \%$ de sa valeur moyenne enregistrée entre 1992 et 2005. Une comparaison de l'évolution des prix des différents produits sur les mêmes périodes apporte des premières nuances au constat d'un embrasement général et sans précédent des prix. Parmi les composants de l'indice du FMI, le pétrole a connu la plus forte hausse (+ $210 \%)$, loin devant les produits alimentaires (+ $39 \%$, boissons incluses) et les produits agricoles à usage industriel (+ $10 \%)$. Si l'on remonte plus avant dans la comparaison des prix des composants de l'indice, la hausse

Tableau 1. Pondération des matières premières dans I'indice de prix du FMI

\begin{tabular}{|lr|}
\hline Indice agrégé & $\mathbf{1 0 0}$ \\
\hline Energie & 63,1 \\
Pétrole & 53,6 \\
Gaz naturel & 6,9 \\
Charbon & 2,6 \\
Hors énergie & 36,9 \\
Produits alimentaires & 18,5 \\
Produits agricoles industriels & 7,7 \\
Métaux & 10,7 \\
\hline
\end{tabular}

Produits alimentaires : Céréales, huiles et tourteaux, produits de l'élevage, produits de la mer, sucre, boissons (café, cacao, thé)

Produits agricoles industriels: Bois, coton, laine, caoutchouc, cuir

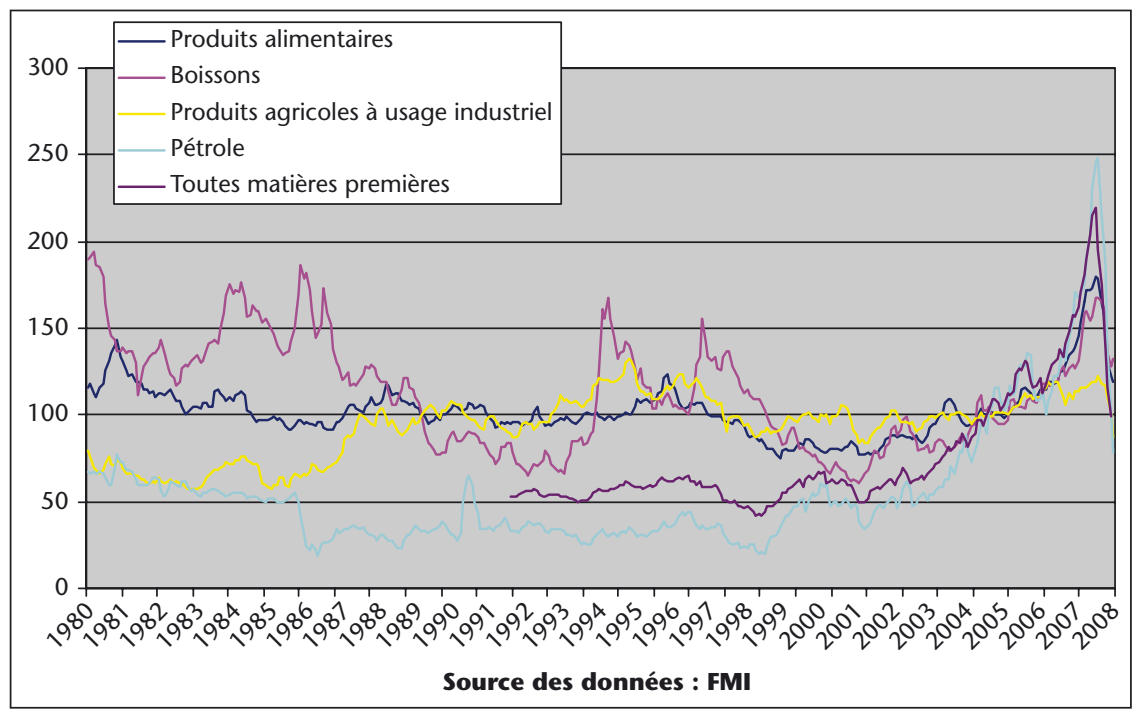

Figure 1. Indices mensuels des prix courants des matières premières $(2005=100)$

moyenne de 2006-2008, comparativement aux valeurs moyennes prises entre 1980 et 2005, s'établit à un niveau comparable pour le pétrole (+ $209 \%)$, contre tout juste $30 \%$ pour les produits alimentaires (boissons incluses) et $22 \%$ pour les produits agricoles industriels. Cette hausse somme doute modeste des prix des matières premières hors énergie signale à quel point la hausse des cours de ces produits a été ponctuelle, n'occupant qu'une séquence très brève de la période 2006-2008. On a pu lire, et retenir ainsi, que les prix du maiis avaient presque triplé entre janvier 2005 et juin 2008, tandis que les prix du blé s'élevaient de $127 \%$, les prix du riz de $170 \%$, les prix de I'huile de soja de $192 \%$ (Mitchell, 2008). Ces variations sont exactes mais elles renseignent sur la hausse ponctuelle entre deux mois particuliers; elles s'estompent si l'on considère des hausses moyennes, calculées sur la base de plusieurs mois ou de plusieurs années.

L'évolution de l'indice du FMI corrigé de l'inflation apporte des nuances supplémentaires au constat d'une hausse sans précédent tant par l'ampleur que par la durée ${ }^{2}$. Les prix réels du pétrole se sont ainsi accrus de $150 \%$ entre la période (1980-2005) et la période (20062008), contre $5 \%$ pour les produits alimentai-

\footnotetext{
2 Plusieurs « déflateurs » permettent de corriger une série de prix ou d'indices mesurés en termes courants. Nous avons choisi l'indice des prix des produits manufacturés (Manufacture Unit Value ou MUV) calculé par la Banque mondiale, indice parmi les plus fréquemment utilisés pour la transformation des prix mondiaux courants en prix réels, c'est-à-dire en prix corrigés de l'inflation.
}

res, tout juste $1 \%$ pour les produits agricoles industriels, les boissons quant à elles enregistrant même un repli (figure 2).

Dans une perspective longue, la hausse des cours des matières premières, toujours considérée en termes réels, a atteint durant le pic de 2006-2008 à peine les deux tiers de la hausse enregistrée en 1974, et un niveau tout juste comparable à celui des pics de 1976 et du second choc pétrolier. La figure 3 montre que la hausse n'atteint pas la moitié pour les produits agricoles.

\section{Le mouvement conjoint des prix des différentes matières premières}

Les écarts dans l'ampleur des hausses de cours des différentes matières premières, considérés dans une perspective longue, contreviennent en apparence à l'idée que ces produits constituent un groupe homogène - les « commodities »- dont les différents composants seraient chacun affectés par les secousses survenus chez un autre, comme les membres d'un même corps en mouvement. L'hypothèse d'une contamination des hausses et des baisses de prix entre marchés de matières premières, ce que dans le jargon des économistes l'on nomme le «co-mouvement» ou «mouvement conjoint des prix » resurgit dans les discussions scientifiques et les débats politiques à chaque « boom » ou « krach » observé sur ces marchés. Que les prix montent ou baissent, une question stratégique que posent les décideurs publics aux économistes porte en effet sur le risque, ou l'opportunité, de voir un tel mouvement de cours persister et se propager 
à d'autres marchés de produits que ceux sur lequel il est observé à l'origine. La hausse du prix du baril à compter de 2005 précédant celle des autres matières premières a ainsi pu suggérer que celles-ci avaient suivi de conserve un mouvement amorcé sur le marché du brent. À nouveau, il convient de distinguer un déplacement conjoncturel des cours à la hausse dont la concomitance entre matières premières est manifeste sur les courbes durant la séquence 2006-2008 -, du mouvement de longue durée.

Une manière simple d'établir la corrélation entre les prix de différents marchés est de recourir au calcul du coefficient du même nom. Le coefficient de corrélation, d'une valeur comprise entre 0 (absence de corrélation) et 1 (corrélation parfaite), indique la proportion de variations de prix commune à deux séries temporelles. Nous l'avons calculé sur la base de l'indice mensuel du FMI, par grande famille de produits, et pour différentes périodes (figure 4).

Nos calculs montrent une corrélation presque parfaite des prix alimentaires et des prix du baril entre 2006-2008, sans aucun antécédent durant les périodes précédentes. Cela est vrai également des boissons ; les produits agricoles à usage industriel ont conservé à l'occasion de cette crise un niveau de corrélation élevé et à peu près identique à celui mesuré durant les dix années précédentes. En 2006-2008, la corrélation entre prix de matières premières est ainsi irréfutable, et d'autant plus spectaculaire qu'elle s'observe sur des marchés dont l'évolution comparée des cours était des plus contradictoires, sinon des plus anarchiques, quelque vingt ans auparavant.

Peut-on parler de co-mouvement pour autant ? Dans le langage commun, à l'évidence oui. L'économiste pour autant ne se satisfait pas d'une corrélation pour établir le comouvement. Après tout, allègue-t-il, deux séries peuvent être temporairement corrélées sans qu'aucune cause commune n'explique une telle corrélation. Un artefact statistique peut ainsi donner l'illusion que deux séries de prix sont dépendantes quand elles ne le sont pas en réalité - la corrélation est alors fortuite. Affirmer l'existence d'un mouvement conjoint requiert de trouver une explication commune à la similitude des mouvements mesurés.

Les économistes formulent le problème de la façon suivante. Les prix des matières premières s'établissant en fonction du coût de leur stockage, lequel dépend du taux d'intérêt, et de l'anticipation de la demande, qui elle dépend de l'activité, une hypothèse raisonnable devrait être que les mouvements conjoints de prix sont provoqués par les variables communes régissant le comportement des stockeurs et de la demande anticipée : taux d'intérêt, PIB, inflation. Aussi le mouvement conjoint des prix

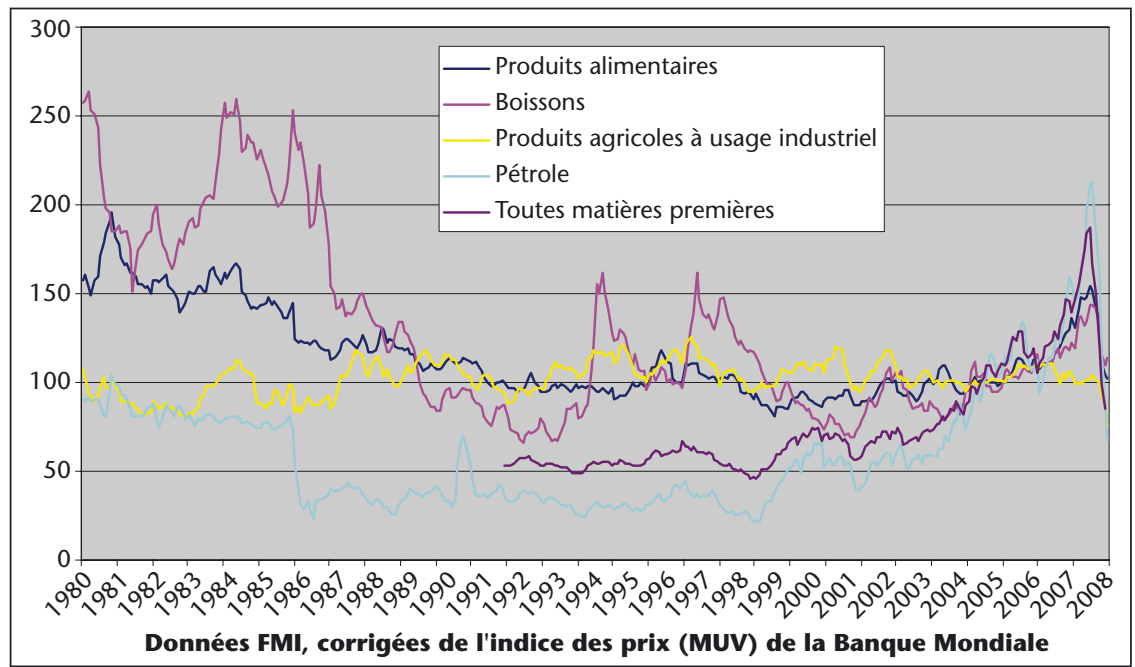

Figure 2. Indices mensuels des prix réels des matières premières $(2005=100)$

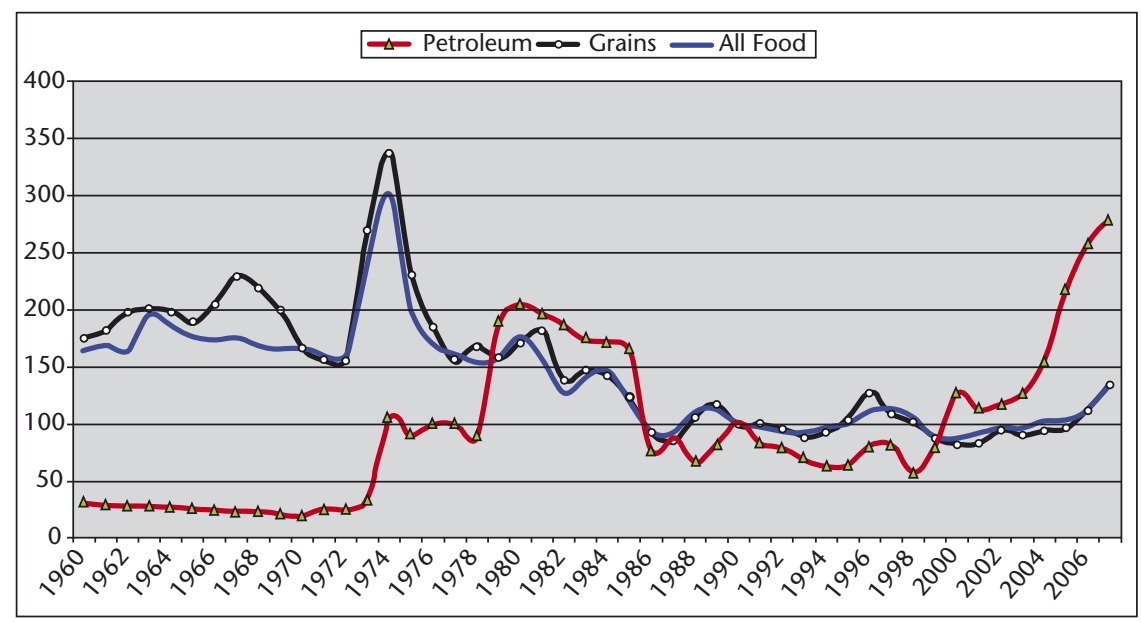

Figure 3. Évolution de l'indices des prix réels (en dollars constants de 1990, base $100=1990$ ), du pétrole, des céréales et de l'ensemble des produits alimentaires

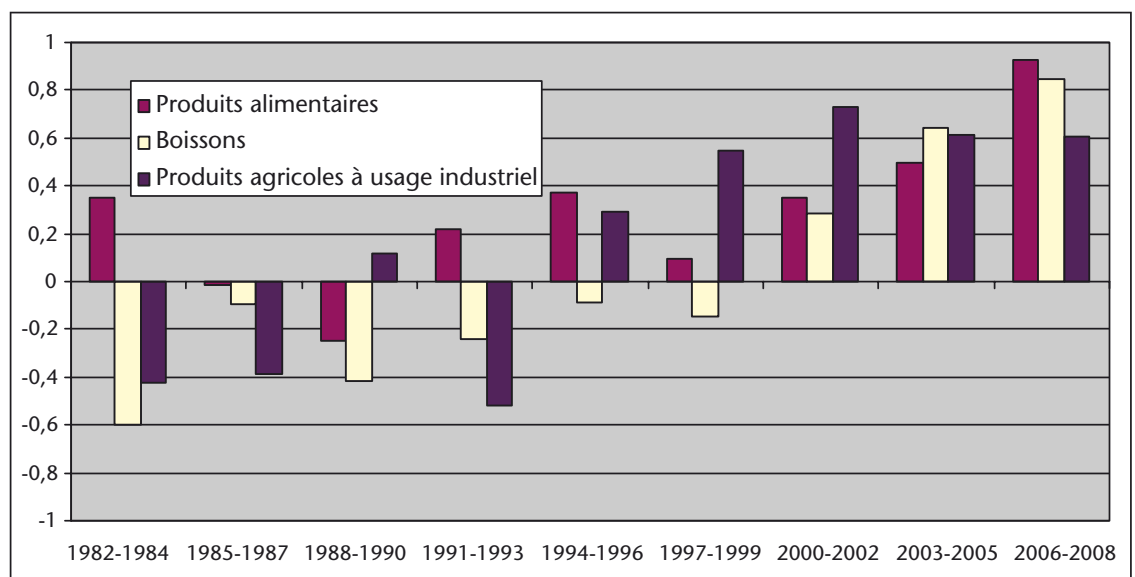

Figure 4. Corrélation des prix courants des matières premières avec les cours du pétrole. Calculs de l'auteur sur la base des indices mensuels publiés par le FMI. 
s'explique-t-il essentiellement, depuis une formalisation due à Pyndick et Rotemberg [2] par des variables macro-économiques. En creux du modèle explicatif de Pyndick et Rotemberg on trouve l'intuition que les mouvements conjoints non expliqués par les variables macroéconomiques seraient dus à la spéculation. C'est ce que les deux auteurs suggèrent après avoir testé leur modèle sur des séries réelles. Si les variables macroéconomiques expliquent une proportion non nulle des fluctuations de prix des matières premières, une partie significative semble être induite par d'autres éléments que les «fondamentaux » (sensés être mesurés par les variables macroéconomiques) et en particulier la spéculation :

"A possible explanation for it is that commodity price movements are to some extent the result of 'herd' behaviour in financial markets. (By 'herd' behaviour we mean that traders are alternatively bullish or bearish on all commodities for no plausible economic reason.) Indeed, our finding would be of little surprise to brokers traders, and others who deal regularly in the futures and cash markets, many of whom have the common belief that commodity prices tend to move together. Analyses of futures and commodity markets issued by brokerage firms, or that appear on the financial pages of newspapers and magazines, refer to copper or oil or coffee prices rising because commodity prices in general are rising, as though increases in those prices are caused by or have the same causes as increases in wheat, cotton, and gold prices"(Pyndick et Rotemberg, 1990, p. 1173).

L'hypothèse de mouvements conjoints de prix non expliqués par les fondamentaux du marché - et donc imputables à la spéculation - a suscité polémiques et réactions ${ }^{3}$. Dans un article au titre explicite - « The Myth of Comoving Commodity Prices " - Cashin, McDermott et Scott [3] avancent ainsi des résultats qui valident I'hypothèse de fluctuations de prix causés par des déterminants macroéconomiques, et en toute logique, invalident l'hypothèse contraire selon laquelle la spéculation prédominerait: "The results carry an important policy implication, as the study provides no support for earlier claims of irrational trading behavior by participants in world commodity markets." Les leçons essentielles que l'on peut tirer de ces polémiques d'économètres est que le mouvement conjoint de prix est à la fois observable et avéré, mais en partie inexpliqué, les parts relatives prises par le hasard, les «fondamentaux » du marché et les variables « hors fondamentaux » (supposées être spéculatives) restant délicates à mesurer. Enfin, notre revue bibliographique montre que I'hypothèse de Pyndick et Rotemberg n'a pas été testée sur des chroniques de prix couvrant la séquence complète de la crise - les travaux les plus récents sur le sujet remontant à 2006. On retiendra donc la définition «commune » et non pas économique au sens strict des mouvements conjoints de prix : les prix des produits agricoles et des produits énergétiques ont connu des mouvements très fortement corrélés durant la période 2006-2008, contrairement à ce que l'on avait pu observer durant les précédentes années.

\section{Pourquoi les prix des matières premières ont-ils augmenté ?}

La répartition entre causes "fondamentales » et causes "spéculatives » des mouvements des prix des matières premières n'est qu'une partie des questions dont se sont préoccupés les acteurs publics et privés concernés durant l'épisode de hausse de trois années. II n'existe que peu d'organismes ou d'institutions qui ne se soient fendu d'une note ou d'un texte d'analyse dans lequel les causes de la hausse des cours sont recensées ${ }^{4}$. Les acteurs de la société civile - syndicats, professionnels, ONG - n'ont pas été en reste, le nombre de tribunes et de prise de parole sur le sujet dans la presse s'élevant à mesure que les cours progressaient.

Plusieurs conclusions peuvent être tirées de ces contributions. La première est qu'une liste de causes candidates plutôt restreinte peut être dressée a posteriori, qui rassemble l'essentiel des explications avancées sans que les diverses institutions ou acteurs qui les ont formulées soient d'accord avec la totalité d'entre elles. La seconde conclusion est que la pondération de ces causes, et donc la mesure de leur contribution relative au phénomène observé, est

${ }^{3}$ Citons Palaskas et Varangis [4], Cashin, McDermott et Scott [3], Ai, Chatrath et Song [5] et Frankel [6].

${ }^{4}$ Citons à titre d'illustration, et pour la seule année 2008, les contributions de la Banque Mondiale (Mitchell, [7]), de la Farm Foundation (Abbott, Hurt, Tyner [8]), de l'IFPRI (Von Braun, [9]), de la FAO (Alexandratos, 2008), de I'USDA (Trostle [10]), de Chatham House (Evans [11]), du Conseil Economique, Social et Environnemental français (Guyau [1]). Signalons aussi le numéro spécial de la revue Agricultural Economics (vol. 39), avec les contributions de Masters et Shively [34], Headey and Fan [35], Dewbre, Giner, Thompson, Von Lampe [36], Flugie [37]. Soulignons enfin le travail de Boussard, Gérard et Piketty [38] qui, sur la base d'un modèle d'équilibre général calculable dynamique incluant le risque et des anticipations imparfaites, expliquent la hausse de la volatilité des prix agricoles par les seules erreurs $d^{\prime}$ anticipation. Parce que ce modèle explique la hausse de la volatilité et non la hausse des prix en tant que telle, nous ne l'avons pas retenu dans notre analyse. Le lecteur curieux pourra se reporter en plus de l'article sus-mentionné, à l'ouvrage le plus récent des auteurs - Boussard, Gérard, Piketty [39]. lacunaire. Le possible est connu, le réel l'est bien moins. Enfin, bon nombre des causes ont été soumises par les mêmes organismes ou personnalités dans des situations de marché totalement opposées, en particulier durant la dépression des cours survenue au tournant du siècle passé. À phénomène inverse, causes hypothétiques identiques. Conservatrice en ce sens, la crise de 2006-2008 a comme confirmé chacun dans l'opinion qu'il se faisait des vertus et des vices de l'économie de marché.

\section{Dix causes hypothétiques}

La hausse des cours peut être lue avec une marge confortable d'erreur comme le résultat d'un déficit de la demande sur l'offre. Les raisons sous-jacentes à la croissance de la demande et à l'inertie de l'offre varient selon les auteurs; néanmoins, une série de huit facteurs affectant I'une ou l'autre, à laquelle il convient d'ajouter deux facteurs complémentaires qui ne leur sont pas directement liés, peut être établie comme suit ${ }^{5}$ :

1. La hausse des prix de l'énergie augmente les coûts de production, et in fine, les prix agricoles. Ses implications sur le coût de l'électricité, du transport, des intrants chimiques, se combinent par une hausse des coûts de production agricole. Des estimations de I'USDA entreprises dans le cadre de la revue régulière des coûts de production par cette institution [12], montrent que la hausse des coûts de production du maïs, du soja et du blé aux États-Unis entre 2002 et 2007 se situe entre 25 et $32 \%$, dont environ $12 \%$ seraient imputables à la hausse des prix de l'énergie. Les prix à l'exportation, qui incluent le coût du transport domestique, auraient subi une hausse de 15-20\% en raison du renchérissement de l'énergie. Mesuré à l'importation (cif) et non plus à l'exportation (fob), la part de l'énergie prise dans les prix agricoles est plus élevée encore, l'indice international des prix du fret ayant été multiplié par dix entre 2002 et le pic de mai 2008 (figure 5) ${ }^{6}$. Nous verrons plus bas que les estimations de I'USDA restent disputées, sinon contestés; la contribution des prix de l'énergie à la hausse des prix agricoles - autrement dit, à une direction particulière de prix - est plus aisée à établir

\footnotetext{
${ }^{5}$ Liste établie sur la base des études, rapports ou compte rendus de conférences de la note 4 supra, en plus des contributions de Gilbert (2008a, 2008b) et de Mili (2008).

${ }^{6}$ L'indice de prix de référence des activités de transport maritime en vrac de matière sèche - dit aussi " fret sec »- est le Baltic Dry Index (BDI), créé à Londres en 1998 et établi sur une moyenne des prix pratiqués sur 24 routes mondiales de transport de matières telles que les minerais, le charbon, les métaux, les céréales et les engrais.
} 


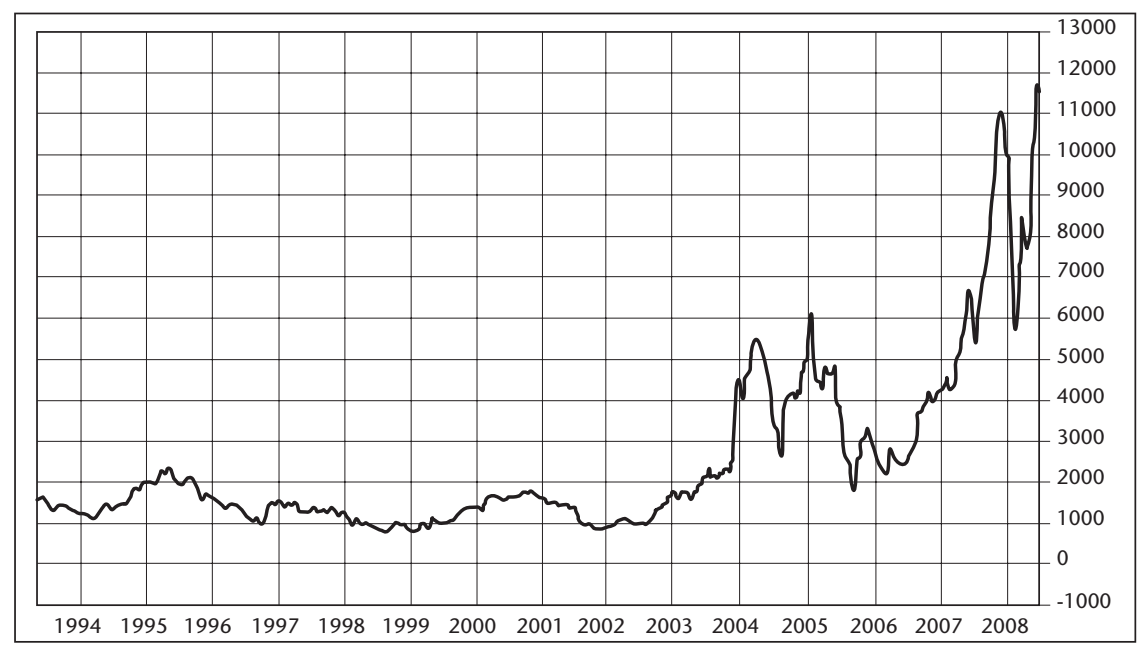

Figure 5. Évolution de l'indice international du prix du fret sec (Baltic Dry Index)

que la contribution au niveau atteint in fine par ceux-ci ${ }^{7}$.

2. La hausse de l'offre de biocarburants et en particulier d'éthanol à partir de maïs nord-américain, a réduit la production agricole disponible à des fins alimentaires. On sait aujourd'hui que la croissance spectaculaire de la production de maïs aux États-Unis entre 2004 et 2007 a été accaparée à $70 \%$ pour la production d'éthanol. Les implications sur les cours mondiaux du maïs, puis en cascade, sur les cours de substituts (tant du côté de l'offre que de la demande) tels que le soja, le blé, puis le riz et les oléagineux et enfin les huiles végétales sont établies, avec la même réserve que celle avancée plus haut: contribuer à la hausse n'est pas équivalent à provoquer une flambée. La canne à sucre brésilienne se voit attribuer un effet inverse à celui du maïs, par la mise à disposition d'une quantité considérable de sucre sur le marché mondial consécutivement à la hausse de la production intérieure de canne destinée pour plus de moitié à la production de carburant. L'offre croissante d'éthanol de canne brésilien aurait ainsi eu un effet d'atténuation déterminant sur le prix mondial du sucre.

3. Les aléas climatiques. L'Australie a été frappée par deux sécheresses consécutives en 2006 et 2007, tandis que cette dernière année, l'Europe subissait des pluies abondantes, avec un même résultat de réduction de l'offre mondiale de céréales. Cette sécheresse historique a eu également un impact sur l'élevage (ovin) néo-zélandais. Le désordre climatique mondial

\footnotetext{
${ }^{7}$ Ce constat, et cette précaution, valent pour toutes les causes hypothétiques avancées ici. Expliquer pourquoi un prix monte est une chose; expliquer pourquoi il atteint des sommets aussi élevés est beaucoup plus compliqué.
}

des années 2006 et 2007 a aussi affecté les plantations de palmier et de caoutchouc indonésiennes et malaysiennes (inondations), les bananeraies antillaises (cyclone Dean) et les rizières birmanes (cyclone Nargis). Mitchell [13] observe cependant que dans le cas des céréales, les mauvaises récoltes australiennes, européennes et ukrainiennes ont été plus que compensées en 2007 par les excellentes récoltes de l'Argentine, du Kazakhstan, de la Russie et des États-Unis. La production mondiale de céréales a bel et bien décliné en 2006 ( $1,3 \%$ seulement) mais elle s'est accrue en 2007 (+ 4,7\%). La météo, toute seule, ne peut expliquer la hausse des cours ; elle a été, dans des régions particulières, un facteur aggravant. 4. La croissance de la demande des pays émergents, et en particulier de la Chine. Energivore et protéinivore, la croissance chinoise à 2 chiffres a vu ce pays se transformer en importateur net de produits qu'il exportait moins de dix ans auparavant. La Chine est aujourd'hui le premier consommateur et importateur de minerais et métaux, le deuxième consommateur de pétrole, le premier importateur mondial de soja et d'oléagineux, de coton, de laine et de caoutchouc. La Chine et I'Inde restent des exportateurs nets de céréales, la démonstration de leur responsabilité dans la hausse des cours de ces produits n'est donc pas triviale, même si les exportations et les stocks ont décliné au fil du temps en raison de la hausse de la consommation intérieure.

5. Les politiques publiques de restriction à l'exportation, mises en place en particulier sur le riz, ont accru l'écart entre la demande et

\footnotetext{
${ }^{8}$ L'Argentine, I'Inde, le Kazakhstan, le Pakistan, I'Ukraine, la Russie et le Vietnam, ont mis en place des restrictions à l'exportation de céréales en 2007 pour atténuer la hausse des prix intérieurs.
}

I'offre mondiale disponibles sur un marché de surcroît déjà étroit - 7 \% de la production mondiale de riz seulement fait l'objet d'un commerce international ${ }^{8}$.

6. La baisse du dollar. Le dollar s'est déprécié de $35 \%$ vis-à-vis de l'euro entre janvier 2002 et juin 2008. Des estimations de la sensibilité des prix des matières premières (libellés en dollars) aux évolutions du dollar (dite encore « élasticité ») montrent qu'une dépréciation de $1 \%$ de la monnaie américaine se traduit par un renchérissement des cours du pétrole de $1 \%$, et un renchérissement moyen des cours des matières premières situé entre $0,5 \%$ et $1 \%$. L'USDA relativise ces chiffres en précisant que la dépréciation du dollar a été beaucoup plus faible vis-à-vis des monnaies asiatiques qu'elle ne l'a été vis-à-vis de l'euro. L'estimation médiane de l'effet de la baisse du dollar sur les cours des matières premières par Mitchell (2008) situe celui-ci aux alentours de $20 \%$.

7. La restructuration des marchés (baisse des stocks). La transformation des filières alimentaires durant les dix dernières années, marquée par une segmentation accrue en aval entre produits transformés, une multiplication des filières spécialisées et accroissement de la dispersion géographique de la production et de la consommation, s'est accompagnée d'une réduction marquée des stocks mondiaux (que ceux-ci se rencontrent dans les pays importateurs ou exportateurs), et particulièrement des stocks chinois. Les stocks mondiaux de céréales (mesurés en pourcentage de la consommation mondiale) ont atteint en 2008 le niveau historique de $15 \%$, le dernier précédent connu étant celui des années 1972/73, précédant la hausse concomitante des prix agricoles et de l'énergie. À la différence de 1973 toutefois, les stocks ont atteint leur niveau le plus bas pendant voire après la crise, et non avant, contribuant donc moins à la provoquer qu'à l'aggraver. Par ailleurs, les faibles niveaux atteints en 2008 sont à replacer dans la perspective longue de stocks historiquement élevés durant toute la décennie 1990 (figure 6). Abbott, Hurt, Tyner [8] soulignent le comportement particulier de la Chine durant cette période. Les stocks chinois de maïs, de blé et de riz s'établissaient à $105 \%$, $90 \%$ et $70 \%$ respectivement en $1998 / 99$. Les stocks étaient tombés à $26 \%$, $38 \%$ et $30 \%$ pour ces trois produits en $2004 / 05$. Si elle n'a pas provoqué la crise, la baisse des stocks depuis 2000 a considérablement réduit les capacités de réaction de l'offre à la hausse des prix.

8. L'étroitesse des marchés. Les produits agricoles dont la hausse fut la plus précoce et la plus

\footnotetext{
${ }^{9}$ Rapport annuel de la Banque des règlements internationaux (BRI), 30 juin 2008.
} 


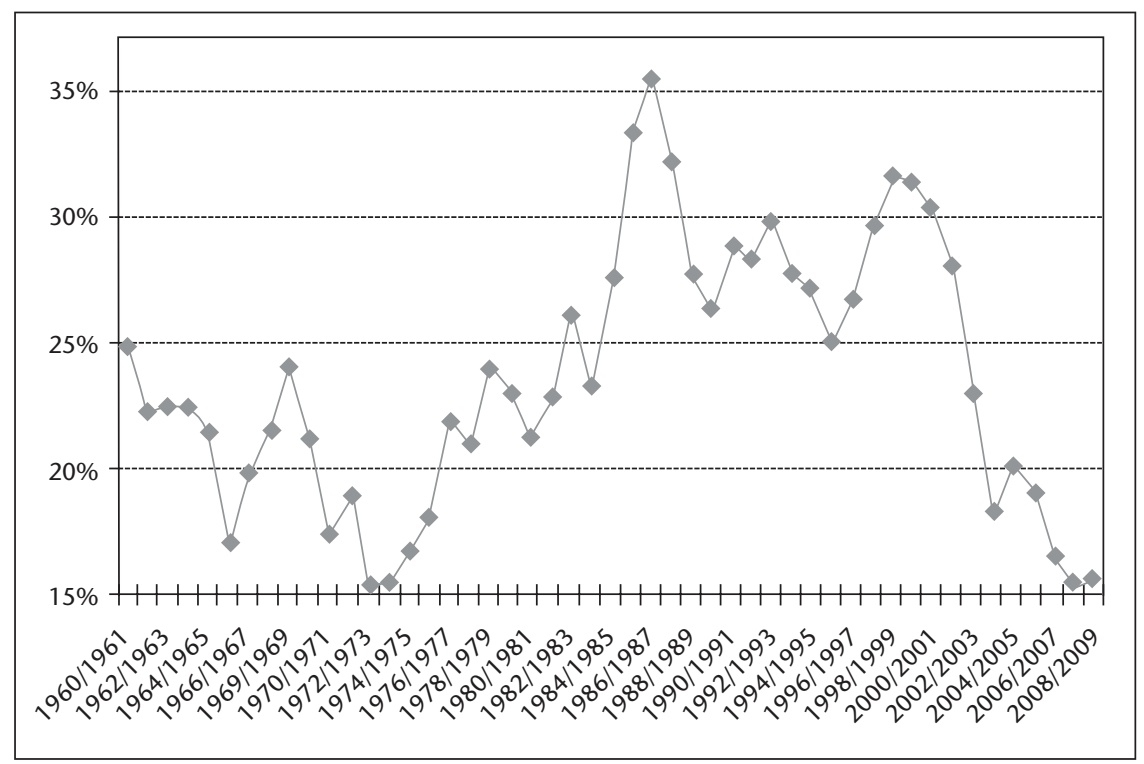

Figure 6. Évolution des stocks mondiaux de céréales (exprimés en pourcentage de la consommation mondiale)

marquée (céréales) sont relativement peu échangés dans le monde en proportion de I'offre disponible : sans tomber au niveau extrêmement faible du riz que nous avons évoqué plus haut, les céréales dans leur ensemble sont essentiellement consommées sur leur lieu de production ( $11 \%$ de la production mondiale de céréales fait l'objet d'un commerce international). La situation est inverse pour les boissons tropicales dont la hausse des cours fut beaucoup moins marquée. L'effet mécanique sur les prix d'un « choc » survenant sur un marché étroit - que ce choc affecte l'offre ou la demande - est intuitif : une variation même faible de la demande ou de l'offre en proportion de la consommation ou de la production mondiale peut devenir significative en proportion des échanges, du fait même que ceux-ci représentent une part ténue des volumes produits ou consommés. En vertu de la loi des grands nombres, un marché «large » absorbera davantage les chocs qu'un marché étroit ${ }^{10}$. L'élément de polémique porte sur les raisons de l'étroitesse de certains marchés agricoles. Hormis les mesures de restriction aux échanges, prises pour l'essentiel pendant la crise et non avant, des mesures de protection ou de subvention ont été incriminés. Les mesures de protection restreignent l'étendue du marché mondial librement accessible. Les mesures de subvention dépriment les prix et dissuadent des offreurs potentiels et efficaces

\footnotetext{
${ }^{10}$ Sous réserve que les «chocs » soient indépendamment distribués et n'affectent pas toutes les régions productrices ou consommatrices en même temps. Un choc météo répond à ce critère.
}

$d^{\prime}$ investir dans la production et d'entrer sur le marché. C'est sur la base de ces deux arguments controversés que Pascal Lamy par exemple, directeur général de I'OMC, a pu au plus fort de la crise presser les pays membres de son organisation de hâter la signature d'un accord de libéralisation ${ }^{11}$.

9. La spéculation. L'influence des marchés à terme sur l'évolution des cours des matières premières au comptant ( « spot ») est une antienne qui s'invite à chaque crise dans le débat public. Plusieurs éléments expliquent qu'en 2006-2008 la « spéculation » et les « spéculateurs » aient été particulièrement mis à contribution pour expliquer I'emballement des cours. Le premier tient à la superposition de deux crises - celles des matières premiers et des subprimes nord américains et à la possible propagation de l'une à l'autre, les marchés de matière première en plein boom offrant un refuge aux investisseurs financiers et l'opportunité d'effacer une partie de leurs dettes et créances douteuses. La seconde raison réside dans la modification de la régulation des marchés à terme de matière première, autorisée par l'administration Bush, afin précisément de faciliter les prises de position sur les marchés à terme de matières premières par des investisseurs sans rapport avec ces marchés. Pour autant, peu d'analyses isole la spéculation comme facteur déclenchant, celle-ci étant en

\footnotetext{
${ }^{11}$ Rapport au Conseil Général de I'OMC du 7 mai 2008, reproduite sur le site de l'OMC http://www. wto.org/french/news_f/news08_f/gc_chair_tnc_7may08_f.htmsous le titre « M. Lamy dit que la crise alimentaire rend encore plus urgente la conclusion du Cycle ».
}

général considérée comme l'écume de la vague qui a soulevé les marchés pendant trois ans. Nous revenons sur les controverses entourant le rôle de la spéculation dans la partie suivante.

10. Le sous-investissement dans le secteur agricole. « La crise alimentaire mondiale est avant tout le résultat de l'insuffisance des investissements observée depuis un certain temps, et qui a causé la baisse de la productivité agricole des pays en développement. Cette baisse a ellemême été aggravée par la dégradation des sols. La crise n'est donc pas causée par un groupe, un pays, ou un type de culture en particulier », affirmaient Kathleen Abdalla et Aslam Chaudhry, de la Division du développement durable du Département des affaires économiques et sociales des Nations Unies (DESA), et John Pender de I'Institut international de recherche sur les politiques alimentaires ${ }^{12}$. Cette idée, formulée surtout au sein des agences de l'ONU et des centres publics de recherche, est sans doute moins polémique que les précédentes. La Banque mondiale elle-même a produit en 2007 un rapport sur le développement dans le monde dont le thème était l'agriculture, en sorte de mea culpa pour le peu d'intérêt que l'institution avait manifesté pour ce secteur, des années durant, dans ses activités de financement. Pour autant, aucune institution ne désigne le peu d'investissements publics dans la recherche agricole comme un facteur déclenchant de la crise.

\section{Des responsabilités relatives difficiles, sinon impossibles à établir}

Aucune étude n'a tenté d'identifier le poids relatif de tous ces facteurs au sein d'un même modèle $^{13}$. L'explication la plus couramment avancée est que chacun a contribué à la hausse des cours: certains de manière autonome, $d^{\prime}$ autres facteurs qui n'ont fait qu'en renforcer l'effet, d'autres de manière conditionnelle et dépendante des autres facteurs énoncés. Autrement dit, certains facteurs ont provoqué la hausse, d'autres l'ont amplifiée. Sur la base de ce constat partagé, les divergences apparaissent, certains auteurs identifiant une variable comme "déterminante", d'autres comme simplement «exagératrice » de la

\footnotetext{
${ }^{12}$ Voir le communiqué de presse de l'ONU « L'insuffisance d'investissements dans la productivité agricole identifiée comme un des principaux facteurs de déclenchement de la crise actuelle ", http:// www.un.org/News/fr-press/docs/2008/ Conf080506-AGRICULTURE.doc.htm

${ }^{13}$ Une exception est celle de Gohin et Dronne [14] dont le modèle toutefois ne parvient pas à expliquer, comme le signalent les auteurs eux-mêmes, près de la moitié des mouvements de prix observés.
} 
hausse des cours et conditionnelle à l'apparition de causes plus lourdes d'effets.

Les variables candidates "déterminantes » sont le plus souvent la hausse des coûts de production agricoles en raison de la cause des prix de l'énergie, l'offre de biocarburants, la hausse de la demande chinoise et la spéculation. Précisons que s'il fait peu de doutes qu'elles sont à même, individuellement, d'amplifier une hausse des prix, leur responsabilité initiale et leur rôle relatifs dans l'ampleur atteinte par l'inflation des cours des matières premières sont encore à ce jour fort mal établis. De manière plus générale, les auteurs soulignent que le poids relatif des différents facteurs dans la hausse des prix alimentaires est difficile, sinon impossible, à mesurer $[10,12]$.

À l'opposé des 4 variables « déterminantes »ou supposées telles - que nous venons de citer, la contribution du taux de change et des politiques de restrictions aux exportations (postérieures à la crise pour la quasi-totalité d'entre elles) semble pouvoir être classée comme conditionnelle, sans trop de polémiques. Enfin, entre ces deux groupes de causes ou facteurs explicatifs, les aléas climatiques, le sousinvestissement public et privé dans le secteur agricole et la restructuration des marchés (baisse des stocks) apparaissent dans une situation intermédiaire. Moins polémiques que le premier groupe de facteurs, ils ne font cependant pas l'unanimité (tableau 2).

II convient de noter que les facteurs dont le poids relatif est le plus controversé sont ceux affectant à la fois les deux marchés qui nous intéressent ici - le marché des produits agricoles et celui de l'énergie. Parmi ceux-ci, nous revenons dans la partie suivante sur les facteurs avec ce que l'on avait pu observer par le passé, les deux catégories de produits, créant l'éventualité d'un lien inédit et persistant à I'avenir entre ces deux marchés. Sur ce double critère de causalité commune et nouvelle (ou renforcée), nous insisterons donc en particulier sur le rôle des biocarburants, de la transition qui auraient lié entre eux, sans comparaison

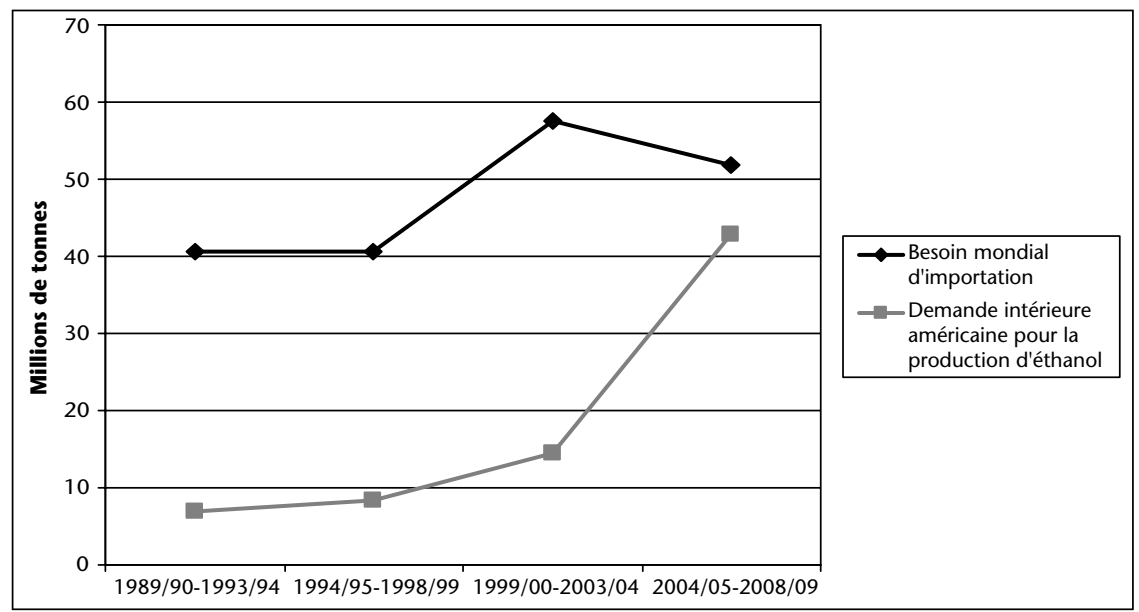

Figure 7. Évolution de la demande d'importation de mais et de la demande intérieure des États-Unis pour la production d'éthanol énergétique et alimentaire des pays émergents, et sur la spéculation.

\section{Les facteurs conjoints de hausse, nouveaux ou renforcés}

\section{Les biocarburants sont-ils coupables?}

Comme dans un célèbre roman de Faulkner, l'intrigue se noue autour d'un épi de maïs. La nouveauté du lien entre marchés de produits agricoles et marchés de l'énergie est difficilement contestable. La hausse du besoin mondial d'importation de maïs a été concomitante à celle de la hausse de la demande intérieure de maïs aux États-Unis pour la production d'éthanol, qui se situe aujourd'hui à environ $29 \%$ de la consommation de maïs de ce pays (figure 7 ). Comme le souligne Collins [15], I'expansion de la consommation de maïs pour la production d'éthanol s'est véritablement accélérée à partir de 2004/05, avec la promulgation aux ÉtatsUnis de I'Energy Policy Act, en période de hausse marquée des cours du baril, avec
Tableau 2. Une tentative de classement des facteurs explicatifs de la hausse des cours. La qualification de controverses « fortement » ou « faiblement controversées » est évidemment subjective.

\begin{tabular}{|lll|}
\hline $\begin{array}{l}\text { Causes fortement } \\
\text { controversées }\end{array}$ & $\begin{array}{l}\text { Causes faiblement } \\
\text { controversées }\end{array}$ & $\begin{array}{l}\text { Causes conditionnelles } \\
\text { relativement bien acceptées }\end{array}$ \\
\hline $\begin{array}{l}\text { Hausse des coûts de production } \\
\text { agricole en raison de la hausse } \\
\text { du prix de l'énergie } \\
\text { Hausse de l'offre de biocarburants }\end{array}$ & $\begin{array}{l}\text { Aléas climatiques } \\
\text { Restruction des marchés (baisse du dollar } \\
\text { des stocks) }\end{array}$ & $\begin{array}{l}\text { Politiques de restriction } \\
\text { aux exportations }\end{array}$ \\
$\begin{array}{l}\text { Croissance de la demande } \\
\text { des pays émergents } \\
\text { Spéculation }\end{array}$ & $\begin{array}{l}\text { Sous-investissement } \\
\text { dans le secteur agricole }\end{array}$ & \\
\hline
\end{tabular}

Source : Auteur l'objectif explicite d'accroître la part des biocarburants dans le «mix » énergétique du pays. Marchés agricoles et marchés de l'énergie sont donc liés par l'anticipation d'un prix élevé du baril et la nécessité de sécuriser l'approvisionnement énergétique des ÉtatsUnis.

On retrouve pareille préoccupation en Europe dans le « paquet climat-énergie » adopté par le Parlement européen le 17 décembre 2008, lequel stipule que chaque État membre de I'Union doit atteindre l'objectif de $10 \%$ de biocarburants dans la consommation d'énergie finale par les transports à l'horizon 2020. Cet objectif implique que $60 \%$ des huiles végétales consommées en Europe le seront pour la production de biocarburant à cette date (Commission européenne [16]). Les données rétrospectives et de prospectives rassemblées par Goldman Sachs [17] montrent qu'il y a eu un «choc » de la demande énergétique adressée aux produits agricoles à compter de 2004 (figure 8), et l'anticipation légitime que la hausse de l'offre de biocarburant devrait persister (figure 9).

Si I'on considère l'ensemble des céréales, des estimations de I'USDA montrent que la demande de maïs américain pour la production d'éthanol entre pour $1 / 3$ dans la croissance de la demande mondiale de ces produits (contre un quart pour la consommation animale et environ $45 \%$ pour la demande alimentaire). Certains ont vu dans ce chiffre des raisons de relativiser la responsabilité de l'éthanol de maïs américain dans la hausse des cours mondiaux du blé et d'autres céréales - et in fine dans la hausse des cours agricoles considérés dans leur ensemble.

L'utilisation de données agrégées au niveau mondial minore immanquablement la part qu'occupe statistiquement la consommation 


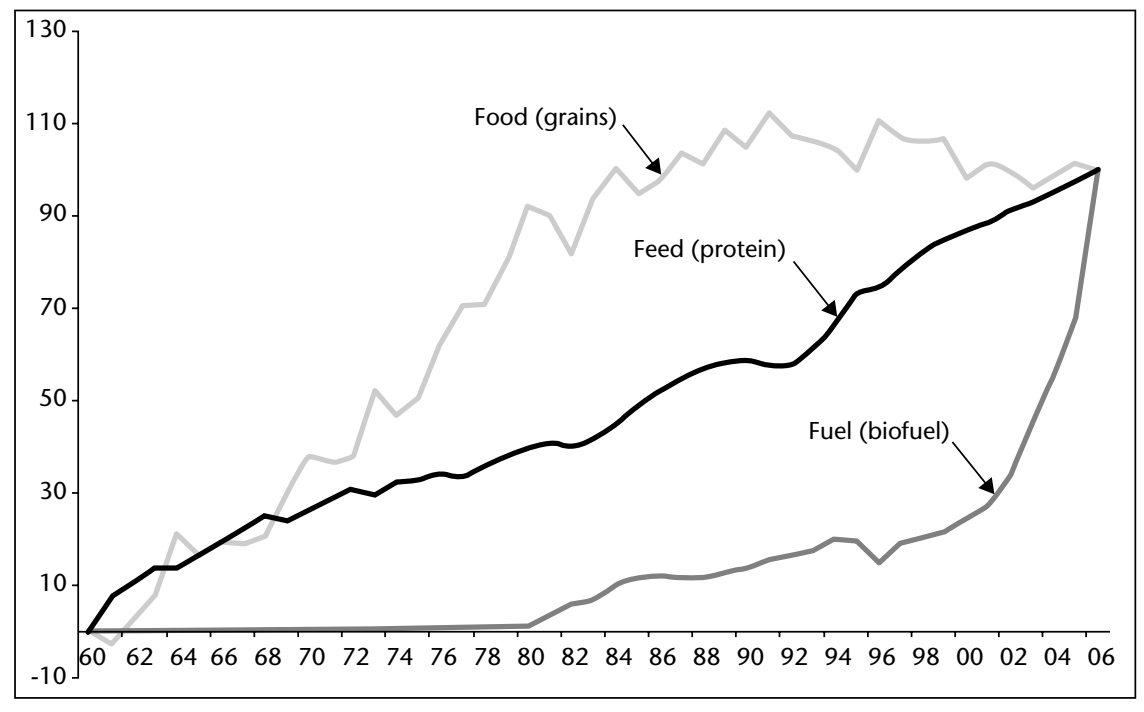

Figure 8. Évolution de l'indice de la consommation par tête de produits agricoles $(2006=100)$

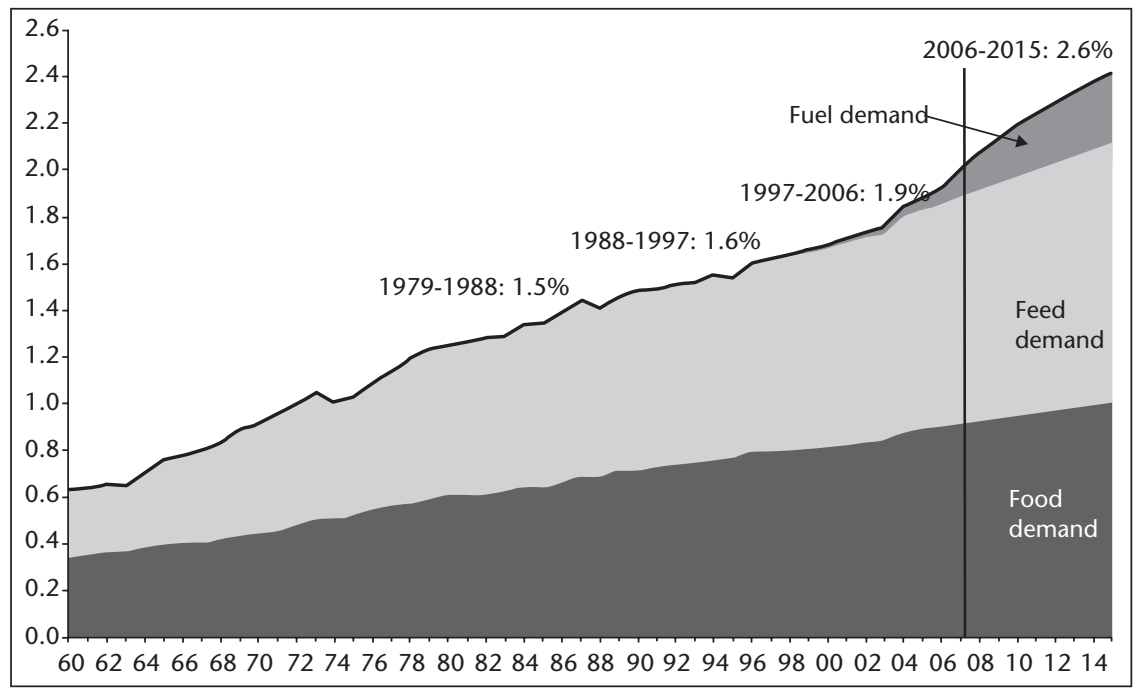

Figure 9. Évolution de la demande agrégée de produits agricoles (milliards t)

énergétique de maïs nord américain dans l'ensemble des céréales produites et consommées (figure 10). Ainsi selon Bricas et Bru [18], "[c]e ne sont pas les volumes actuels de produits agricoles destinés aux agrocarburants qui expliquent la hausse des prix, sauf localement chez les fournisseurs de maïs des États-Unis, principaux producteurs de ces carburants. D'après les estimations d'Agrimonde, projet de prospective mené par le Cirad et I'Inra, sur le total des calories végétales produites dans le monde, moins de $5 \%$ sont destinées aux usages non alimentaires dont font partie les agrocarburants. L'alimentation humaine représente $55 \%$ de ce total et l'alimentation animale $30 \%$. En outre, les volumes destinés aux agrocarburants sont, pour le moment, faibles. Ainsi, selon l'Agence internationale de l'énergie, en 2005, 1 \% des terres cultivées servaient à produire des agrocarburants remplaçant contribution de la hausse de l'offre de biocarburants de maïs américain dans l'accroissement des prix mondiaux agricoles durant l'épisode 2006-2007 (tableau 3).

Retenons aussi que la tension provoquée par la raréfaction réelle et anticipée du maïs alimentaire sur le marché nord-américain a eu des effets directs sur les cours du soja et du blé, par substitution dans les assolements, laquelle a contribué à la hausse et à l'amplitude exceptionnelle des cours, même si la mesure exacte de cette contribution est impossible, tout comme sa mesure relative, en l'état des modèles mondiaux disponibles.

\section{La transition alimentaire et énergétique des pays émergents}

La croissance des pays émergents, en particulier de I'Inde et de la Chine, est couramment citée comme une hausse majeure des cours alimentaires ${ }^{14}$. Comme le rappelle Alexandratos [19], la voix d'Amartya Sen s'est mêlée au concert, pour souligner que « les riches avaient de plus en plus faim » et que leur appétit risquait $d^{\prime}$ être fatal aux plus pauvres ${ }^{15}$. Des mesures par la FAO signalent pourtant que la croissance de la consommation chinoise et indienne de céréales a été sensiblement moins forte entre 2002 et 2008 que durant les années 1990 - exception faite du maïs [19]. Et si la consommation de viande a continué de croître, la croissance s'est plutôt ralentie durant les cinq dernières années. L'évolution de la consommation de viande n'a pas eu non plus le caractère exceptionnel que l'on a pu lire dans la presse. Au total, l'effet de la hausse du revenu chinois et indien sur les prix mondiaux des céréales est modeste tout au plus selon la FAO et la Banque mondiale [22].

Tel n'est pas le cas en revanche des oléagineux et des huiles végétales, dont la demande $d^{\prime}$ importation indienne mais surtout chinoise s'est considérablement accrue depuis le tournant du siècle (figure 11). La Chine par exemple importe aujourd'hui $45 \%$ des graines de soja échangées dans le monde, contre à peine $10 \%$ il y a dix ans. La transition alimentaire vers des régimes de consommation riches en lipides survient dans les pays émergents à des niveaux de revenu beaucoup plus faibles que ce que I'on a pu observé au XIX $X^{\mathrm{e}}$ et $\mathrm{X} \mathrm{X}^{\mathrm{e}}$ siècle parmi les pays de l'OCDE [23]. Par ailleurs, la demande d'oléagineux pour la production de biodiesel a également joué un rôle non négligeable. L'USDA estime que $7 \%$ de l'offre d'huiles végétales dans le monde étaient utilisés en biodiesel en

\footnotetext{
${ }^{14}$ Voir par exemple par exemple le World Economic Outlook du FMI (p. 60) d'avril 2008.

15 "The Rich Get Hungrier", New York Times, 28 mai 2008
} 


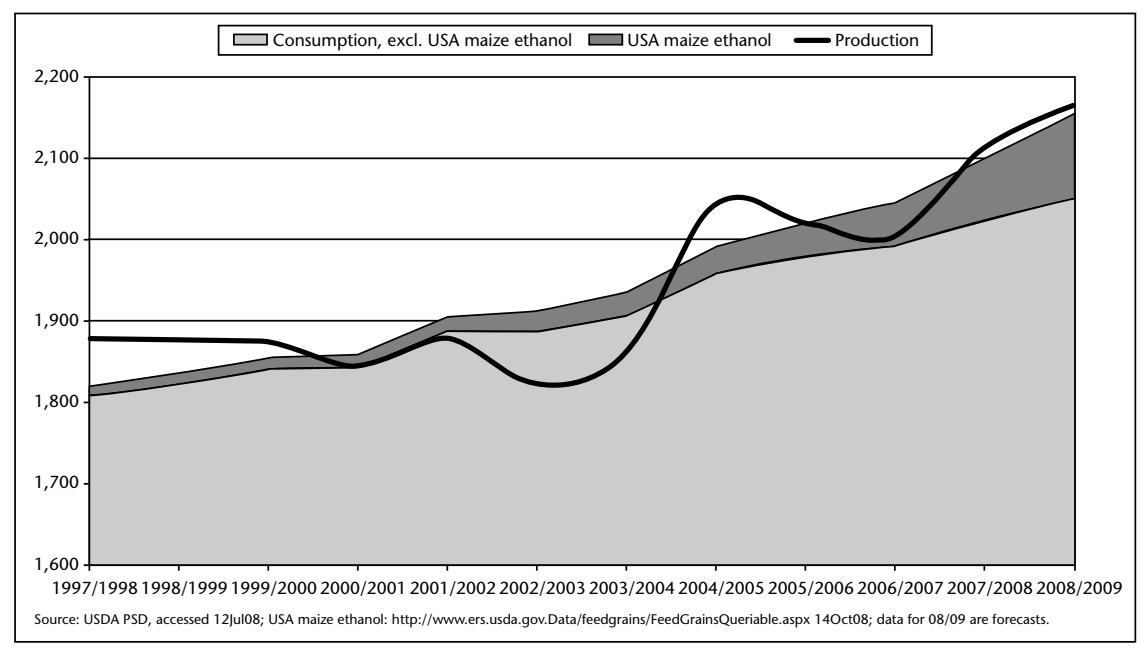

Figure 10. Évolution de la consommation mondiale de céréales

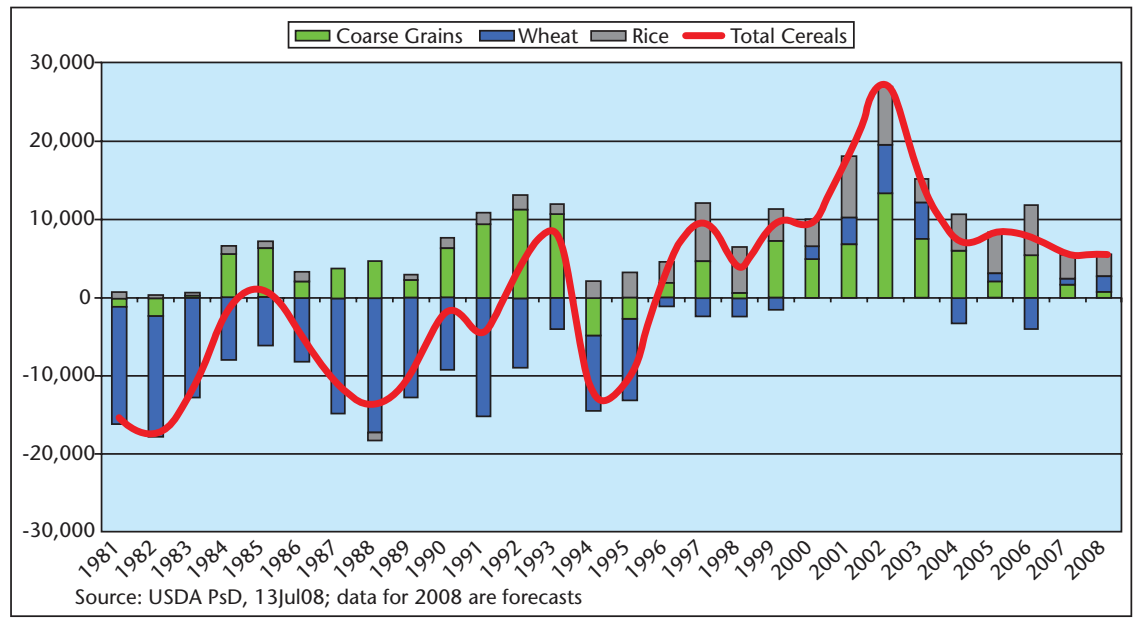

Figure 11. Commerce net de céréales de l'Inde et de la Chine (1 000 t)

2007, lequel entrerait pour $1 / 3$ dans la croissance de la consommation d'huiles végétales entre 2004 et 2007. L'effet revenu a donc très vraisemblablement joué pour ces produits, tant sur la consommation énergétique que sur la consommation alimentaire, sans pour autant créer un «choc » qui une année particulière

Tableau 3. Estimations de la propagation des effets de la hausse de la production de biocarburant de mais américain sur les cours des céréales

\begin{tabular}{|ll|}
\hline Auteurs & $\begin{array}{l}\text { Hausse expliquée } \\
\text { Produits }\end{array}$ \\
\hline Lipsky [20] & $70 \%$ Maïs \\
& $40 \%$ Soja \\
Collins [15] & $60 \%$ Maïs \\
Rosegrant [21] & $47 \%$ Maïs \\
& $26 \%$ Blé \\
& $25 \%$ Riz \\
\hline
\end{tabular}

Tableau 4. Pondération des indices de matières premières (S\&P-GSCl et DJ-AIG)

\begin{tabular}{|c|c|c|c|}
\hline & & $\begin{array}{l}\text { S\&P- } \\
\text { GSCI }\end{array}$ & DJ-AIC \\
\hline \multirow{9}{*}{$\begin{array}{l}\text { Produits } \\
\text { agricoles }\end{array}$} & Cacao & 0,2 & 0 \\
\hline & Café & 0,5 & 2,7 \\
\hline & Maïs & 3,6 & 6,9 \\
\hline & Coton & 0,7 & 2,2 \\
\hline & Huile de soja & 0 & 2,9 \\
\hline & Soja & 0,9 & 7,4 \\
\hline & Sucre & 2,1 & 2,8 \\
\hline & Blé & 3 & 3,4 \\
\hline & Blé KC & 0,7 & 0 \\
\hline \multirow[t]{2}{*}{ Élevage } & Porc & 0,8 & 2,5 \\
\hline & Bœuf & 1,6 & 4,1 \\
\hline \multirow[t]{6}{*}{ Énergie } & Pétrole brut brent & 14,8 & 0 \\
\hline & Pétrole brut WTI & 40,6 & 15 \\
\hline & Diesel & 5,4 & 0 \\
\hline & Fuel domestique & 5,3 & 4,5 \\
\hline & Essence & 4,5 & 4,1 \\
\hline & Gaz naturel & 7,6 & 16 \\
\hline \multirow{5}{*}{$\begin{array}{l}\text { Métaux } \\
\text { de base }\end{array}$} & Aluminium & 2,1 & 6,9 \\
\hline & Plomb & 0,2 & 0 \\
\hline & Nickel & 0,5 & 1,7 \\
\hline & Zinc & 0,4 & 1,8 \\
\hline & Cuivre & 2,6 & 6,7 \\
\hline \multirow{2}{*}{$\begin{array}{l}\text { Métaux } \\
\text { précieux }\end{array}$} & Or & 1,5 & 6,1 \\
\hline & Argent & 0,2 & 2,4 \\
\hline
\end{tabular}

Source : Standard \& Poor, Dow Jones, Masters et White [24]

marchés physiques (les physical hedgers dits encore commercial en référence à une activité de commerce de matière première) et les spéculateurs sur matière première (ou « non commercial ») n'intervenant eux jamais sur le marché physique, les marchés à terme de matières premières ont connu un bouleversement considérable ces cinq dernières années. Alors que prédominaient les opérateurs physiques et que l'intervention des spéculateurs, nécessaire à l'octroi de liquidités dans le marché, restait limitée et sévèrement réglementée (et ce depuis les leçons de la crise de 1929 prises par l'administration Roosevelt en 1936 dans son Commodity Exchange Act), la distinction entre marchés à terme de matières premières et marchés financiers est devenue poreuse. Un assouplissement de la législation en 1991, 1998 puis en 2003, a ouvert les marchés à terme de matières premières à un nombre croissant $d$ 'investisseurs extérieurs, les fonds de pension et compagnie d'assurance-vie étant les plus connus du grand public. Tel est le cas en parti- 
culier des investisseurs sur indice de matière première (index speculators), investisseurs qui voyaient dans la liberté accrue d'intervenir sur les bourses de matière première l'opportunité d'enregistrer des gains supérieurs aux rendements sans risque, tout en diversifiant leur portefeuille par des placements sur des actifs présentant une corrélation faible ou négative avec d'autres classes d'actifs.

Les indices de matières premières les plus connus sont le The Standard \& Poors - Goldman Sachs Commodity Index, le the Dow Jones - AIG Commodity Index, I'indice Deutsche Bank liquid commodity index (DBCl) et l'indice Reuters CRB. Les principales différences entre ces différents indices portent sur les pondérations accordées à certains types de matières premières, en particulier les produits énerla pondération du S\&P-GSCI (lequel comporte 24 commodities) et de l'indice DJ-AIG (19 produits). L'évolution de cet indice sert de référence aux placements des investisseurs institutionnels sur ces marchés. En pratique, les gétiques. Le tableau 4 montre la composition et

investisseurs sont «long» c'est-à-dire acheteurs sur les marchés à terme de matières premières. Ils spéculent à la hausse en faisant tourner (« rouler » en anglais) une position d'achat qu'ils dénouent avant maturité et renouent de période en période, enregistrant des profits substantiels tant que les cours des composants de l'indice montent. Le sort des prix de l'énergie et des prix des denrées se trouve ainsi, par l'activité de ces spéculateurs sur indice, intrinsèquement lié.

Entre 2003 et 2008, les achats sur marchés à terme de matières premières effectués sur la base d'indices tels que ceux que l'on vient de décrire sont passés de 13 à 317 milliards de dollars. Au cœur de l'argument de Masters et White figure le fait que la part des positions prises sur les marchés à terme par spéculateurs sur indice a connu un essor sans précédent entre 2005 et 2008 (figure 12). En 1998, les physical hedgers ou « commerciaux » détenaient $77 \%$ des positions d'achat prises sur les marchés à terme de matières premières, les spéculateurs traditionnels (« non commercial ») $16 \%$ et les

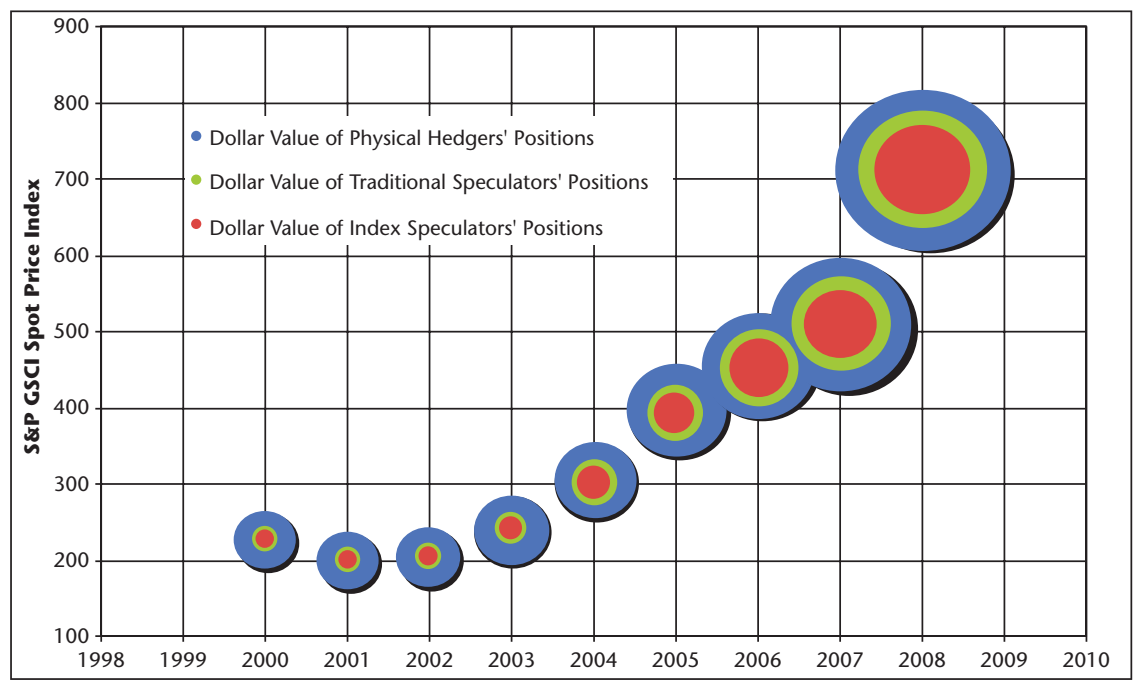

Figure 12. Valeur en dollars des positions prises sur les marchés à terme par les différents intervenants - commerciaux (" physical hedgers »), non commerciaux traditionnels (" traditional speculators») et spéculateurs sur indice.

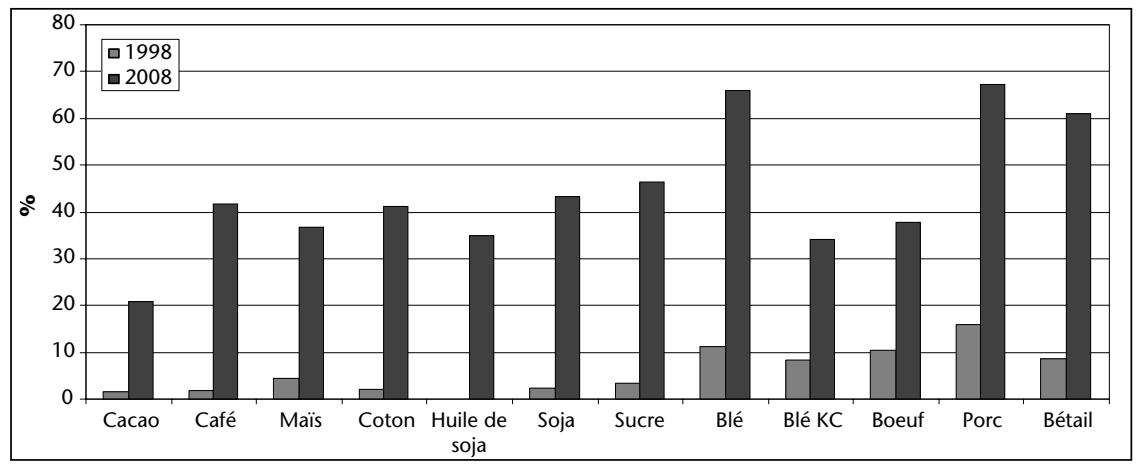

Figure 13. Proportion des positions d'achat («long ») prises par les investisseurs sur indice spéculateurs sur indice $7 \%$. En 2008, les proportions se sont renversées. Les opérateurs physiques détiennent $31 \%$ des positions d'achat, les spéculateurs traditionnels $28 \%$ et les spéculateurs sur indice $41 \%$. Cette proportion peut approcher $70 \%$ sur certains produits (figure 13). Ce sont 2,7 millions de contrats à terme de matières premières qui ont été achetés par les spéculateurs sur indice entre 2003 et 2008, contre 1,4 millions pour les spéculateurs traditionnels et moins de 900000 pour les hedgers.

Plusieurs études revues par Mongars et Marchal-Dombrat [25] dans un document publié la Banque de France, examinent I'hypothèse de matières premières constituant une classe d'actifs à part entière, sous-jacente à I'argumentation de Masters et White. Parmi les études recensées, celles de Gorton et Rouwenhorst [26] et JP Morgan [27] suggèrent que l'introduction des matières premières dans un portefeuille diversifié permet d'améliorer le ratio rendement/risque du portefeuille. Ibbotson Associates [28] situent la part optimale des matières premières au sein d'un portefeuille diversifié dans une fourchette de $22 \%$ à $29 \%$. "L'intérêt des investisseurs pour les matières premières s'est intensifié au cours des dernières années en liaison avec la progression spectaculaire des cours de la plupart d'entre elles » notent Mongars et Marchal-Dombrat en 2006. "Certains investisseurs institutionnels - tels que des fonds de pensions néerlandais ou californiens - ont confirmé qu'ils s'étaient constitués ou envisageaient de se constituer une exposition modeste (moins de $5 \%$ de leurs actifs) aux matières premières. En parallèle, le développement de nouveaux supports d'investissement a permis aux investisseurs particuliers d'acquérir également ce type d'exposition. Le sentiment que la rapide croissance économique en Asie devrait se poursuivre et se traduire par une demande soutenue de ces pays pour les matières premières peut expliquer cet engouement. Celui-ci semble être également alimenté par les travaux académiques et d'analystes de marché soulignant que l'introduction des matières premières dans les portefeuilles est une source appréciable de diversification des risques. Ce constat et cette interprétation suggèrent que les investisseurs sont amenés à introduire progressivement mais durablement les matières premières dans leurs portefeuilles ».

Peut-on néanmoins affirmer que les matières premières constituent une classe d'actifs à part entière ? S'interrogent les deux auteurs. Au terme de leur étude, ils apportent "une réponse positive à cette question dans la mesure où, sur longue période, le rendement des placements liés aux matières premières paraît supérieur au rendement sans risque et présenter une corrélation faible ou négative avec d'autres classes $d^{\prime}$ actifs et ne semble pas pouvoir être reproduit à 
partir d'une simple combinaison linéaire d'actifs».

La seule incertitude concerne à nouveau la causalité réelle entre hausse et ampleur des cours d'un côté, et spéculation excessive de l'autre. Masters et White soutiennent une telle causalité, qu'ils établissent sur la base d'une corrélation significative entre positions d'achat spéculatives et hausses des prix des matières premières. Ils soulignent par ailleurs, avec d'autres auteurs, que la contamination, par les activités sur indice, d'une spéculation particulière propre au marché du pétrole, est à peu près avérée [29], tandis que les présomptions d'un report de positions spéculatives sur les matières premières après effondrement de la bulle internet (figure 14), puis des bulles de l'immobilier et enfin celle des subprimes est très forte même si elle n'est pas démontrée. La remise en cause du rôle déclencheur de la spéculation est à rechercher du côté d'économistes tels que Irwin et Holt [30] et Gilbert [31]. Les réserves des premiers concernent une période qui ne nous intéresse pas directement ici, puisqu'elle précède la flambée de 20062008. Christopher Gilbert quant à lui a testé économétriquement la responsabilité des investissements sur indices dans la hausse des cours de 4 produits côtés au marché à terme du Chicago Board of Trade - le maïs, le soja, I'huile de soja et le blé. II montre que la causalité est avérée dans le cas du soja - mais dans le cas du soja seulement, concluant ainsi qu'au total, les preuves d'une responsabilité de la spéculation sur indice dans le boom des prix des matières premières entre 2006 et 2008 restent limitées.

Nous pouvons retenir cependant que, même limitée au soja selon les calculs de Gilbert [32], la spéculation a joué un rôle moteur dans la hausse des cours d'une matière première, en lien direct avec la hausse provoquée sur le pétrole par ces mêmes investisseurs institutionnels. Nous avons au total complémentarité de trois causes, chacune déterminante de la hausse du prix d'une denrée conjointement à celui de l'énergie : I'offre de biocarburant dans le cas du maïs, la spéculation sur indice dans le cas du soja, et l'anticipation générale (même si elle est erronée sur la seule base de la balance courante actuelle des produits agricoles des pays considérés) de la demande d'énergie et de produits alimentaires de la part des pays émergents durant les prochaines années.

\section{Conclusion}

Notre travail a consisté à revoir et organiser les facteurs explicatifs de la hausse des cours des produits alimentaires et des produits de l'énergie selon leur degré de responsabilité commune dans l'épisode du «boom » des années 2006-2008, et leur possible implication dans un rapprochement futur de ces deux marchés. Sur la base de notre revue critique de la littérature, sept résultats relativement robustes,

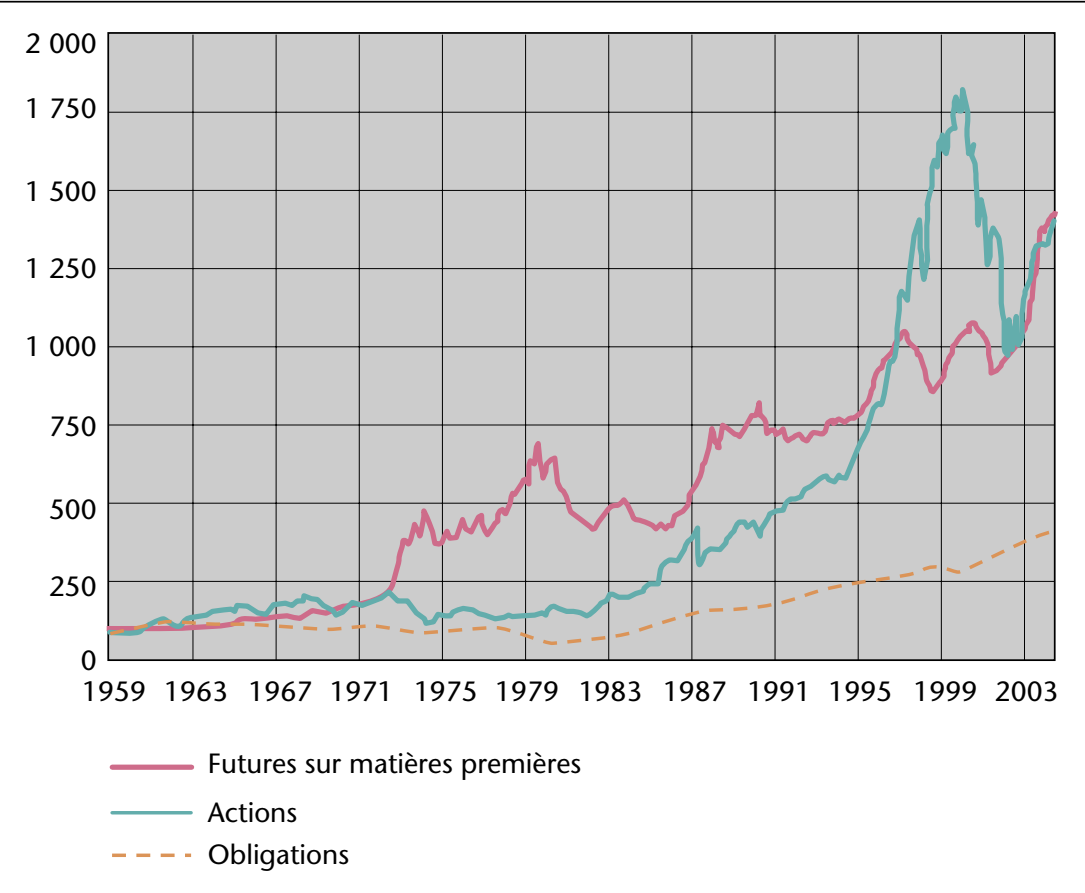

Figure 14. Rendement réel comparé des actions, des obligations américaines et des contrats à terme de matières premières (base $100=$ juillet 1957) même s'ils restent entachés d'une appréciation controversée, méritent d'être relevés.

1. La hausse des cours des matières premières dans leur ensemble est certes spectaculaire lorsqu'on la mesure en dollars ou en euros courants, mais elle est très relative et même modeste en comparaison des sommets atteints durant les années 1970. Elle touche de surcroît les différents produits constituant l'agrégat des matières premières de manière très différenciée - le marché de l'énergie étant le plus affecté par l'envolée des prix, loin devant les produits alimentaires, les boissons et les produits agricoles à usage industriel.

2. Un mouvement conjoint des cours agricoles et de l'énergie se manifeste pendant l'épisode bref de hausse s'étirant de 2006 à 2008, sans antécédent connu durant les vingt années qui précèdent. Il y a bien une nouveauté, au regard de l'histoire récente, dans l'envolée simultanée des cours de I'alimentation et de l'énergie, même si l'épisode est bref.

3. Une série de dix causes hypothétiques, plus ou moins controversées, peut être avancée, qui comporte les éléments suivants: hausse des coûts de production agricole en raison de la hausse du prix de l'énergie, hausse de l'offre de biocarburants, croissance de la demande des pays émergents, spéculation, aléas climatiques, restructuration des marchés (baisse des stocks), sous-investissement dans le secteur agricole, baisse du dollar, enfin politiques de restriction aux exportations. Aucune évaluation quantitative ne permet de dégager les pondérations relatives de ces différentes causes hypothétiques d'évolution à la hausse des prix de marché.

4. Parmi ces causes, les plus controversées sont celles candidates à l'explication de la hausse conjointe des prix alimentaires et de l'énergie. Il s'agit de l'offre de biocarburants, de la croissance de la demande des pays émergents, et de la spéculation.

5. À l'exception de la demande des pays émergents, dont la responsabilité immédiate semble pouvoir être rejetée, les deux autres facteurs de hausse conjointe que sont les biocarburants et la spéculation ont, d'après les évaluations statistiques, joué un rôle déterminant dans la hausse immédiate d'un produit particulier. Il s'agit du maïs dans le cas des biocarburants, et du soja dans le cas de la spéculation.

6. Les substitutions du côté de l'offre physique de produits alimentaires (maïs/blé, maiis/soja, soja/blé) et les substitutions du côté de la demande « papier »-c'est-à-dire financière de ces produits à des fins de diversification de risque (indices de matières première/equity), ont tout ensemble, dans l'anticipation d'une tension croissante et prolongée du marché en raison de hausse attendue de la demande des 
pays émergents, propagé la hausse à d'autres produits agricoles et amplifié celle-ci.

7. Des interrelations inédites ou considérablement renforcées, en comparaison du passé, entre marchés agricoles et marchés de l'énergie se sont au total cristallisées en 2006-2008 autour de l'essor des biocarburants de première génération, de la spéculation sur indice et de l'anticipation d'une demande d'importation de produits alimentaires et énergétiques par les pays émergents à long terme. La persistance de cette interrelation dans un avenir proche semble probable, ces deux types de marchés étant liés "par de nouvelles modalités » selon les conclusions de Good et Irwin [33] - même si une telle persistance est soumise à plusieurs incertitude. Celles-ci concernent pour l'essentiel la vitesse de remplacement des biocarburants de première génération par des biocarburants de seconde génération; les règles encadrant la spéculation sur indice aux ÉtatsUnis susceptible d'émerger durant la gestion de la crise financière par la nouvelle administration américaine ; enfin les trajectoires de croissance énergétique et alimentaires des pays en développement (croissance reposant sur les services, "à l'indienne », ou sur l'industrie, « à la chinoise »).

\section{RÉFÉRENCES}

1. Guyau L. Les marchés des matières premières : évolution récente des prix et conséquences sur la conjoncture économique et sociale. Rapport du Conseil Economique, Social et Environnemental, Les éditions des Journaux officiels. 2008.

2. Pindyck RS, Rotemberg IJ. The Excess CoMovement of Commodity Prices. Economic I $1990 ; 100: 1173-89$.

3. Cashin PA, McDermott Cl, Scott A. The Myth of Co-moving Commodity Prices. Bank of New Zealand Discussion Paper G99/9. 1999.

4. Palaskas TB, Varangis PN. Is There Excess CoMovement of Primary Commodity Prices? Working Paper WPS 758, The World Bank, Washington, DC. 1991.

5. Ai C, Chatrath A, Song F. On the Comovement of Commodity Prices. Am J Agric Econom 2006; 88: 574-88.

6. Frankel J. Comment: Real rates key to commodity prices. Reuters, 19 mars 2008.

7. Mili Y. Controverses sur les determinants de la hausse des prix alimentaire. Mimeo. Paris : IDDRI, 2008.
8. Abbott PC, Hurt C, Tyner WE. What's Driving Food Prices. Issue Report 75. Farm Foundation, 2008.

9. von Braun J. Biofuels, International Food Prices, and the Poor, Testimony to the United States Senate Committee on Energy and Natural Resources, June 12, 2008. Washington, DC. : IFPRI, 2008.

10. Trostle R. Global Agricultural Supply and Demand : Factors Contributing to the Recent Increase in Food Commodity Prices. Report WRS-0801 from the USDA Economic Research Service, version révisée en juillet 2008.

11. Evans A. Rising Food Prices: Drivers and Implications for Development. Chatham House Food Supply Project, CH/BP 08/01. 2008.

12. USDA, 2008

13. Mitchell D. A Note on Rising Food Prices. Policy Research Working Paper 4682, The World Bank,Washington, DC. 2008.

14. Gohin et Dronne (2008).

15. Collins K. The Role of Biofuels and Other Factors in Increasing Farm and Food Prices. Supporting material for a review conducted by Kraft Food Global, Inc. 2008.

16. Commission Européenne. The impact of a minimum $10 \%$ obligation for biofuel use in the EU27 in 2020 on agricultural markets, AGRI G2/WM D. 2007.

17. Currie J. Food, Feed, and Fuels : An outlook on the Agriculture, livestock and biofuel markets", Goldman Sachs International, mars 2007.

18. Bricas, Bru. La hausse des prix alimentaires au Sud: causes, conséquences, propositions, http://www.cirad.fr/fr/actualite/communique. php?id=919) 2008.

19. Alexandratos N. Food Price Surges: Possible Causes, Past Experiences and Relevance for Exploring Long-Term Prospects. Pop Develop Rev 2008 ; 34 : 663-97.

20. Lipsky J. Remarks At the Council on Foreign Relations, New York, Mai 2008

21. Rosegrant M. Biofuels and Grain Prices : Impacts and Policy Responses. Testimony for the U.S. Senate Committee on Homeland Security and Governmental Affairs, 7 May 2008. Washington, D.C. : IFPRI, 2008.

22. Banque Mondiale. Responding to High Food and Fuel Prices Double Jeopardy, Paper for the G-8 Hokkaido-Toyako Summit, July 2, 2008, Washington, DC. 2008

23. Voituriez T. L'huile de palme et son marché : la modélisation de la volatilité. Thèse de doctorat en sciences économiques, Université de Paris I. 1999.

24. Masters MW, White AK. The Accidental Hunt Brothers. How Institutional Investors Are Driving Up Food and Energy Prices. Special Report, 31 juillet 2008.

25. Mongars $\mathrm{P}$, Marchal-Dombrat $\mathrm{C}$. Les matières premières: une classe d'actifs à part entière ? Banque de France, Revue de la stabilité financière, $n^{\circ} 9$, décembre 2006.

26. Gorton $\mathrm{G}$, Rouwenhorst $\mathrm{G}$. Facts and fantasies about commodity futures. NBERWorking Paper, $n^{\circ} 10595$, février 2005.

27. JP Morgan Securities Ltd. Are alternatives the next bubble? Septembre 2006.

28. Ibbotson Associates. Strategic asset allocation and commodities. 27 mars 2006.

29. Masters MW, White AK. The Accidental Hunt Brothers - Act 2. Index Speculators Have Been a Major Cause of the Recent Drop in Oil Prices", special. Update 2008 ; 10 (septembre).

30. Irwin et Holt (2004)

31. Gilbert CL. Commodity Speculation and Commodity Investment. Discussion Paper 20, Universita Degli Studi Di Trento. 2008.

32. Gilbert CL. How to Understand High Food Prices. Discussion Paper 23, Universita Degli Studi Di Trento. 2008.

33. Good D, Irwin S. The New Era of Corn, Soybean, and Wheat Prices, Marketing and Outlook Brief 08-04, Department of Agricultural and Consumer Economics, University of Illinois at UrbanaChampaign, September 2008.

34. Masters WA, Shively G. Introduction to the special issue on the world food crisis. Agricultural Economics 2008 (39) : 373-4.

35. Headey D, Fan S. Anatomy of a crisis: the causes and consequences of surging food prices. Agricultural Economics 2008 (39) : 375-91.

36. Dewbre J, Giner C, Thompson W, Von Lampe M. High food commodity prices : will they stay? who will pay? Agricultural Economics 2008 (39): 393-403.

37. Fuglie KO. Is a slowdown in agricultural productivity growth contributing to the rise in commodity prices? Agricultural Economics 2008 (39) : 431-41.

38. Boussard JM, Gérard F, Piketty MG. Pourquoi les prix agricoles augmentent-ils? OCL 2008 ; 15 (2) : 81-7.

39. Boussard JM, Gérard F, Piketty MG. Should global agriculture be liberalized? Theories, models and realities. Montpellier, France: Quae, 2008. 